\title{
Practice and reflection on the management mode of pain quality control in emergency pre-check and triage
}

\author{
Liping Wang ${ }^{1 \#}$, Chunyan Song ${ }^{2 \#}$, Yunjuan Bai ${ }^{1}$, Xiaoping Huang ${ }^{1}$, Haixia Shi ${ }^{1}$, Jian Pan ${ }^{1}$ \\ ${ }^{1}$ The First Affiliated Hospital Zhejiang University School of Medicine, Hangzhou, China; ${ }^{2}$ Hang Zhou Shu Lan Hospital, Hangzhou, China \\ Contributions: (I) Conception and design: L Wang; (II) Administrative support: C Song; (III) Provision of study materials or patients: Y Bai; (IV) \\ Collection and assembly of data: X Huang; (V) Data analysis and interpretation: H Shi, J Pan; (VI) Manuscript writing: All authors; (VII) Final \\ approval of manuscript: All authors. \\ "These authors contributed equally to this work. \\ Correspondence to: Jian Pan. Emergency Department, The First Affiliated Hospital Zhejiang University School of Medicine, No. 79 Qingchun Road, \\ Shangcheng District, Hangzhou 310003, China. Email: 1509031@zju.edu.cn.
}

\begin{abstract}
Background: Triage decision making is crucial for patients arrived at emergency room as it influences the clinical outcome. Appropriate standard to discriminate patients into different category is challenging. We introduced a suitable emergency preview triage pain model for quality management methods through the pain assessment module.

Methods: The pain quality control and management of emergency pre-examination triage were realized by designing the pain assessment module of the intelligent triage system. Clinical nurses completed the assessment of pain score and PQRST pain content through the pain tool in the pain assessment module of the intelligent triage system. The computer system would automatically assess the triage category according to the pain score. Triage nurses determined the priority of emergency care based on the type of care.

Results: The pain quality control target monitoring reached $95 \%$ of the target management value in ten months. There were two months when the target management value was not reached 95\%, June 2019 (94.28\%), and December 2019 (94.28\%), respectively, slightly lower than the target management value (95\%). Conclusions: The application of the pain quality control management mode of emergency preexamination triage unifies the standards of emergency pre-examination triage pain, standardizes the pain assessment and improves the level of emergency pre-examination triage pain management.
\end{abstract}

Keywords: Pain management; emergency triage; quality control

Submitted Apr 20, 2020. Accepted for publication Jun 04, 2020.

doi: 10.21037/apm-20-1108

View this article at: http://dx.doi.org/10.21037/apm-20-1108

\section{Introduction}

Pain is the cause of emergency treatment for most patients. Almost $75-80 \%$ of patients complain of pain of different degrees (1). Despite the widespread presence of pain, analgesia is still insufficient (2). Among them, the most common reason for inadequate pain control is that there is no correct evaluation of pain (3). Pain is different from vital signs and cannot be measured objectively by instruments. Therefore, the immeasurability of pain and the urgent time setting in the emergency department increases the difficulty of pain assessment, which undoubtedly poses a considerable challenge to emergency triage for nurses responsible for emergency pain management (4). Currently, there are no clinically relevant pain management standards for emergency preview triage.

Based on this, we tried to conduct pain assessment module through the design of intelligent triage system. Apart from that, we established standardization of pain assessment process, dynamic evaluation and reevaluation of tracking implementation of emergency triage personnel access, pain-related knowledge training system 
implantation. We used this triage system to monitor of monthly pain control data quality. The pain triage module is composed of three parts: chest pain, abdominal pain and other pain. The chest pain assessment module is equipped with screening items for high-risk chest pain, which is conducive to the identification of high-risk chest pain and the implementation of green channel management. The abdominal pain assessment module sets up the screening items for acute abdominal diseases, which is helpful to improve the recognition and management of acute abdominal diseases. In each pain assessment module, a variety of subjective pain assessment scales and objective pain assessment scales are implanted. Meanwhile, PQRT rapid triage mode is used to conduct a comprehensive and complete assessment of the contents of pain.

We conducted this study in order to assess emergency patients pain dynamically, comprehensively, accurately and shunt patients rapidly. Moreover, we aimed to help the patients get the diagnosis and treatment as soon as possible, improve engineering emergency triage pain management quality, provide the reference specification for clinical emergency triage nurses pain assessment and management too.

\section{Methods}

\section{Pre-check and triage in pain quality control management}

\section{Design of triage pain assessment module}

Based on the Manchester pre-test triage scale (MTS), the pain assessment module consists of abdominal pain, chest pain, and three other submodules. Furthermore, they were implanted in the three modules including visual analog scale (VAS), numerical rating scale (NRS), facial scale (FACE), language description scale (DPIS), The Face, Legs, Activity, Cry, Consolability Behavioral Scale (FLACC), Critical-care Pain Observation Tool (CPOT). The nurse in the triage chose the proper pain scale to assess the degree of pain on the patients.

PQRST fast model of pain assessment was embedded in other sub-modules, including $\mathrm{P}$ (provokes) cause, nature of the $\mathrm{Q}$ (quality), $\mathrm{R}$ (radiate) radiation area direction, $\mathrm{S}$ degree (severity), $\mathrm{T}$ (time) time five assessment items, to achieve complete assessment in patients with abdominal pain, chest pain and other pain $(5,6)$.

According to the 2016 American college of cardiology (ACC) and the American heart association (AHA) nonST-elevation acute coronary syndrome guide, module pain in chest pain sub-modules added entry of acute chest pain at-risk screening and early warning for high-risk patients with chest pain to realize rapid screening in patients with acute myocardial infarction (MI) and the green channel of the single disease management (7). The sub-module of chest pain consists of three parts: (I) the types of chest pain, such as suspected cardiogenic chest pain, suspected noncardiogenic visceral chest pain, and other chest pain; (II) the associated symptoms (sweating, dizziness, radiation pain, palpitations, shortness of breath); (III) the duration in minutes, hours, days. Furthermore, suspected cardiac chest pain with entries added further screening to the entries, i.e., progression increased myocardial enzymes and electrocardiogram tip, a positive troponin, suspected myocardial infarction, unstable angina, suspected stable angina after nitroglycerin with ease, and unable to alleviate after nitroglycerin was used. The triage nurse just checked the screening items according to the actual situation assessment with the triage system automatically show triage categories. For example, if checking for cardiac chest pain but not checking for any accompanying symptoms of chest pain patients, the system triage categories presented as III. These results indicated that the patient was in a potentially dangerous and challenging situation with relatively stable vital signs and needed treatment as soon as possible. The target response time should be $<30$ minutes. If checking for the cardiac chest pain and any items with symptoms or cardiac chest pain, and the triage system showed I or II class, this suggested that the patient was in a critical condition or critical and a doctor must be informed to take intervention measures and start green channel management process for high risk of acute chest pain.

The pain module adds the screening items for acute abdomen in the abdominal pain sub-module, including accompanying symptoms like vomiting, apparent abdominal distension, constipation, and indigestion or particular types like a history of abdominal aortic aneurysm, related to the last menstrual period or pregnancy. For instance, if checked the abdominal pain with vomiting symptoms abdominal distension or particular type, classification of triage system showed II class, suggesting the patient was in a critical condition and need to notify the doctor for immediately processing and monitoring closely immediately, the target response time should be $<15$ minutes.

The department set up a special pain quality control nurse, and used the pain quality control checklist every month to complete the collection of pain data of 5 pain patients in the form of on-site inspection. At the same time, the nurses in charge of the pain patients were examined for 


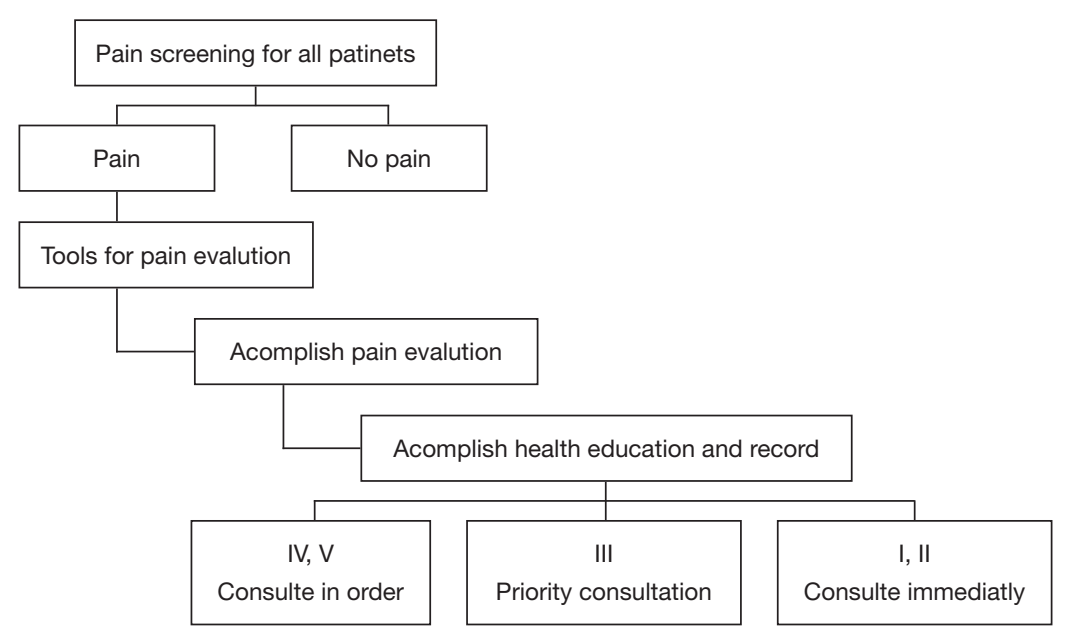

Figure 1 Process of emergency pre-examination triage.

their knowledge of pain. The pain quality control nurse recorded the items that did not conform to the pain quality control, calculated the pain target quality control compliance rate in the form of a percentage, and reported the quality control results to the head nurse. According to the requirements of the nursing department's pain management objectives, the head nurse will give feedback at department meetings, formulate and implement improvement measures, and the pain quality control nurse will continuously track the implementation effect, so as to realize continuous quality improvement of pain management.

When triage nurse was evaluating pain module, for any type of pain score value $=4$ points in patients with acute pain with triage categories as IV class, indicating the patients were in acute but stable condition and triage nurse needed to report attending doctor. If the patient did not seek medical care within 30 minutes, there would be a red alarm in the triage system to remind the triage nurse to evaluate again. For patients with acute pain with score $>4$ and $<7$ points on the pain assessment, triage system classified as III class, indicating a prompt emergency. Then triage nurse needed to arrange priority report doctor visits, the target response time should be $<30$ minutes. For the patients who were assessed as having a pain score $\geq 7$ and classified as II class, indicating critically ill. Triage nurse should immediately report the doctor with immediate treatment and close monitoring, and the target response time should be $<15$ points.

\section{Triage pain assessment process}

Triage nurses handle pain screening and assessment of all patients. Self-reporting is widely regarded as the most reliable yardstick for measuring personal pain, which is to say, self-assessment of pain is the gold standard for pain assessment (8). Triage nurses play an essential role in enabling patients to assess and manage pain to achieve patients' self-assessment of pain (9). In the pre-examination triage desk, we supplied a variety of pain assessment tools such as assessment ruler and pain assessment form for patients' self-assessment of pain. The pain management committee of the hospital has also formulated pain-related propaganda and education prescriptions. The department has formulated simple pain education prescriptions according to the pain characteristics of emergency patients.

\section{Preview triage pain assessment process}

Preview triage nurses should choose the pain assessment tools with reliability and validity according to pain patients' age, education, cognitive ability (10). Meanwhile, the triage nurse was supposed to complete a review of PQRST content fast, accomplish related knowledge education sealed record, arrange the order according to the nursing triage system category. The pain assessment process of emergency pre-examination triage was shown in Figure 1.

\section{Results}

\section{Dynamic pain evaluation and re-evaluation of pain}

Studies have shown that triage nurses screened and assessed pain for the first time patients with pain arrived at the emergency department. But most of them do not reassess the degree of pain. Despite the high degree of pain, they 
still did not get enough analgesia (11). Therefore, in the process of triage, through the intelligent triage system for pain re-assessment and the training of triage personnel, we realized the dynamic assessment and re-assessment of patients and ensured patients to get the corresponding diagnosis and treatment in time. Dynamic evaluation refers to the continuous and dynamic evaluation of the change of pain symptoms and the report of new pain in emergency treatment. According to the new evaluation nursing category, the pre-check triage nurse arranges the patient to see the doctor or takes corresponding treatment measures according to the doctor's advice. Pain assessment refers to the triage nurse again give pain analgesic interventions (including drug and non-drug interventions) to assess pain again, whether the patient needs to confirm the curative effect and intervention. The triage nurse adds a new pain assessment record and prepares related intervention measures in the other column. The pain quality control nurses can query the pain score through the triage system to track the dynamic evaluation and reevaluation of pain.

\section{Triage personnel access and pain-related knowledge training}

Based on the N1-N5 hierarchical management system of the nurses' human resources, the department required the level of nurses involved in triage work should be N3 level or above to ensure the quality and safety of triage. At the same time, the head nurse and the pain quality control nurse conducted the triage system operation training and pain-related knowledge training for the nurses who were admitted to the pre-examination triage. Studies have shown that theoretical courses combined with simulated case exercises will effectively improve the management of pain by emergency triage nurses (12). Based on this, we designed a pain management course combining theory and case simulation. The theoretical part of the course covers the content of pain overview, pain assessment tools, assessment methods, pain records, analgesic methods, adverse analgesic reactions and treatment measures, and health education. In the case simulation exercise, trauma, acute abdomen, highrisk chest pain, cancer pain, and other cases were designed, requiring nurses to carry out correct pain assessment records, re-assessment, and health education for simulated patients through an intelligent triage system.

\section{Monitoring of pain quality control data}

The department set up a particular pain quality control nurse, who shall be responsible for the continuous improvement of pain quality. Specific responsibilities of the pain quality control nurse contained regularly participating in the pain-related knowledge training and meetings organized by the hospital's pain quality control committee. Five pieces of pain quality control data of pain patients were collected, statistically analyzed, and reported to the head nurse and the hospital pain quality control committee by using the self-made self-examination form of pain quality control every month. The training of painrelated knowledge of the new comrades, advanced study, rotation, and pre-admission triage nurses in the department was done. The self-designed pain quality control selfexamination form adopted by the pain quality control nurses is composed of four items. One pain assessment, admission with pain screening, correct selection of pain assessment tools, correct assessment methods, assessment results consistent with the condition, assessment results recorded. Two, the dynamic evaluation and re-evaluation of pain (in patients with acute pain score $=4$ points IV class assessment, record again after 30 minutes. If individuals had a pain score $\geq$ of 4 , they should report to doctors. With a pain assessment score $\geq 7$, immediate treatment is required, record pain management including intravenous administration for 15 minutes, skin, muscle, and rectum medication for 30 minutes, and digestive tract administration for 1 hour (13). Three, pain records, and publicity; there are records of the implementation of pain publicity and education required of pain records, including location, nature, degree, time, and related factors. Four, nurse assessment is done for the selection of commonly used pain assessment tools and assessment methods. The pain quality control nurse collected and collated the pain data every month, recorded the items that did not conform to the pain quality control, and calculated the compliance rate of the pain assessment in the form of a percentage.

\section{Pain monitoring data management}

Goal management is a modern management method that is goal-oriented, people-centered, and results-oriented, enabling organizations and individuals to achieve the best performance, and objectively measure and evaluate the objectives (14). We adopted the method of goal management to check the data of pain quality control and set the target value of pain quality control as $95 \%$. We presented it in the form of a chart, visually displayed the results of monthly pain quality control, and showed the results in the monitoring column of objective management 


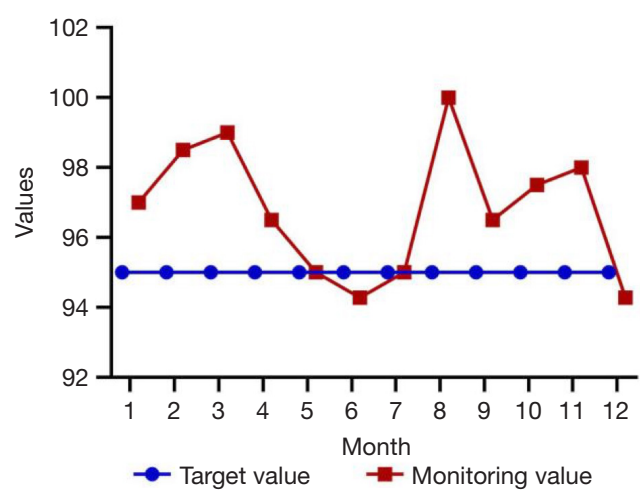

Figure 2 Pain quality control target monitoring data in 2019.

data on the wall of the corresponding nursing unit in the emergency department. According to the monitoring, in 2019, there were two months in which the monitoring of pain quality control target in our department failed to meet the standards, respectively in June (94.28\%) and December $(94.28 \%)$, which were slightly lower than the target management value of $95 \%$. Figure 2 shows the annual monitoring data of pain quality control from January to December 2019. According to the results of data monitoring, the head nurse would analyze and give feedback to the monitoring data in monthly department meetings based on the requirements of the pain target management, formulate and implement improvement measures, and continuously track the implementation effect. Meanwhile, problems in pain quality control, cause analysis, corrective measures, expected goals, and effect evaluation was recorded in the medical care management system. Afterward, each nurse was needed to timely complete the reading and confirmation of nursing quality problems in that month to achieve effective management of pain.

\section{Discussion}

\section{Unify the triage criteria for pain and standardize the pain assessment process}

Unify the triage criteria for pain mainly refers to that the computer system gives a uniform emergency triage category and decides the priority of patients' medical treatment rather than the subjective judgment of triage nurses. Standardization means that clinical triage nurses evaluate each item involved in pain assessment one by one through a unified pain triage module, so as to achieve the completeness of pain assessment. NCCN clinical cancer pain practice guidelines mentioned that patients with the pain score $>7$ received emergency treatment. However, the pain degree has not been included in the pre-test triage indicator system and combined with the triage category. Pain module of triage system unified standard triage patients with pain, pain assessment process, the specification for the preview triage personnel management, the standardization of pain assessment and homogeneity also implemented the screening for all patients pain and confirm the pain of patients after screening or high-risk patients to conduct a comprehensive assessment of the pain (15).

According to the goal requirements of grade A class 3 hospital for pain management, the goal of pain quality control is set at $95 \%$. The hospital has a special pain management committee, which provides training on pain related knowledge and conducts a baseline survey on the quality of pain management in the hospital on a regular basis. Each clinical department is equipped with pain quality control nurses to conduct pain quality inspection every month and record the quality control results.

\section{Dynamic evaluation and re-evaluation of pain to ensure patient safety}

There are certain blind spots in the dynamic evaluation and re-evaluation of clinically acute pain patients (12). Compared with hospitalized patients, patients in the emergency department have mobility, so it is difficult for pre-check triage nurses to realize dynamic evaluation and re-evaluation of patients with acute pain. We built a model for nurses, doctors, patients, and their families to participate in pain quality management, track pain patients, conducted dynamic assessment and reassessment, and improve the quality of pain management to ensure patient safety through the education of pre-check triage nurses, the coordination of diagnosis and treatment between doctors and perfect information system.

\section{Nurses' pain management knowledge promotes pain quality control and management}

Most of the nurses in the clinical practice of pain assessment are not ideal, and the most important reason is the lack of knowledge of relevant pain management (2). Studies have shown that nurses' pain training courses can improve pain assessment and management and improve patient satisfaction (12). We promoted the quality of pre-triage pain management through training in triage system pain 
assessment for triage nurses, theoretical, and pain case simulation exercises. Also, according to the latest pain guidelines and the latest national standards of pain diagnosis and treatment, our pain management course would add relevant course contents, update knowledge and further promote the quality of pain management.

\section{Pain data monitoring to achieve continuous quality management of pain}

Continuous quality improvement is the core of nursing management. Through continuous quality improvement, pain management can be effectively improved (16). The pain quality control nurses collected and sorted out the pain quality control data of emergency pre-examination and triage every month to supply the basis for continuous quality improvement of pain. By continuously monitoring pain data and guided by goal management, we take the compliance rate of pain assessment as the evaluation index of pain quality management, which is helpful to improve the level of pain care management (17).

This study preliminary established an effective emergency preview triage pain quality control management method, also has obtained certain achievements. However, there are some limitations in this study. For one hand, training course is single. For another hand, pain patients with subjective assessment tool for pain assessment module unrealized self-reported direct import function. On top of it, sensitivity and applicability of the quality control check list and patient engagement was lacking.

Therefore, we need to further optimize the triage system and the design of pain training courses in the future. Also, improving the applicability and sensitivity of the evaluation indicators of pain quality is needed to elevate the evaluation of patients' satisfaction with pain management. Based on the information platform, it is the future direction of pain quality control and management to explore the management mode applicable to the pain quality control of emergency pre-examination and triage.

\section{Conclusions}

The effectiveness of pain management is one of the indicators of the quality of care. In this study, a pain quality control and management method of emergency pretest triage were initially set up, and positive results were achieved to some extent, but there were some limitations. Therefore, in the future pain management process, we need to optimize the triage system further and course design, evaluate the applicability and sensitivity of pain quality evaluation indicators and increase the evaluation of patients' satisfaction with pain management. Based on the information platform, it is the future direction of pain quality control and management to explore the pain quality control and management model applicable to emergency pre-examination and triage, realize the standardization and standardization of pain nursing management, and provide quality service for pain patients.

\section{Acknowledgments}

Funding: None.

\section{Footnote}

Data Sharing Statement: Available at http://dx.doi. org/10.21037/apm-20-1108

Conflicts of Interest: All authors have completed the ICMJE uniform disclosure form (available at http://dx.doi. org/10.21037/apm-20-1108). The authors have no conflicts of interest to declare.

Ethical Statement: The authors are accountable for all aspects of the work in ensuring that questions related to the accuracy or integrity of any part of the work are appropriately investigated and resolved.

Open Access Statement: This is an Open Access article distributed in accordance with the Creative Commons Attribution-NonCommercial-NoDerivs 4.0 International License (CC BY-NC-ND 4.0), which permits the noncommercial replication and distribution of the article with the strict proviso that no changes or edits are made and the original work is properly cited (including links to both the formal publication through the relevant DOI and the license). See: https://creativecommons.org/licenses/by-nc-nd/4.0/.

\section{References}

1. Moss D, Willmarth E. Hypnosis, anesthesia, pain management, and preparation for medical procedures. Ann Palliat Med 2019;8:498-503.

2. Effiong A, Kumari P. Integrating palliative care and emergency medicine for optimal management of sickle cell pain in the wake of the United States opioid epidemic. 
Ann Palliat Med 2018;7:274-6.

3. Ferrero S, Vellone VG, Barra F. Pathophysiology of pain in patients with peritoneal endometriosis. Ann Transl Med 2019;7:S8.

4. Giusti GD, Reitano B. Pain assessment in the Emergency Department Correlation between pain rated by the patient and by the nurse An observational study. Acta Biomed 2018;89:64-70.

5. Phillips JL, Heneka N, Hickman L, et al. Can A Complex Online Intervention Improve Cancer Nurses' Pain Screening and Assessment Practices? Results from a Multicenter, Pre-post Test Pilot Study. Pain Manag Nurs 2017;18:75-89.

6. Ciaramelli E, Neri F, Marini L. Improving memory following prefrontal cortex damage with the PQRST method. Frontiers in Behavioral Neuroscience 2015;9:211.

7. 2016 ACC/AHA Guideline Focused Update on Duration of Dual Antiplatelet Therapy in Patients With Coronary Artery Disease A Report of the American College of Cardiology/American Heart Association Task Force on Clinical Practice Guidelines An Update of the 2011 ACCF/AHA/SCAI Guideline for Percutaneous Coronary Intervention, 2011 ACCF/AHA Guideline for Coronary Artery Bypass Graft Surgery, 2012 ACC/AHA/ACP/ AATS/PCNA/SCAI/STS Guideline for the Diagnosis and Management of Patients With Stable Ischemic Heart Disease, 2013 ACCF/AHA Guideline for the Management of ST-Elevation Myocardial Infarction, 2014 AHA/ACC Guideline for the Management of Patients With NonST-Elevation Acute Coronary Syndromes, and 2014 ACC/AHA Guideline on Perioperative Cardiovascular Evaluation and Management of Patients Undergoing Noncardiac Surgery. Circulation 2016;134:e123-55.

8. Kim YS, Park JM, Moon YS, et al. Assessment of pain in the elderly: A literature review. Natl Med J India

Cite this article as: Wang L, Song C, Bai Y, Huang X, Shi H, Pan J. Practice and reflection on the management mode of pain quality control in emergency pre-check and triage. Ann Palliat Med 2020;9(4):1879-1885. doi: 10.21037/apm-20-1108
2017;30:203-7.

9. Price C, Lee J, Taylor AM, et al. Baranowski5Initial assessment and management of pain: a pathway for care developed by the British Pain Society. British Journal of Anaesthesia 2014;112:816-23.

10. Registered Nurses'Association of Ontario Assessment and Management of pain M. 3rded To ronto, ON:Registered N urses' Association of Ontario, 2013:1-105.

11. Ghazali DA, Kenwaya P, Clerya R, et al. A multicenter randomized control trial evaluating professional practice assessment of patient pain management after simulation training course: Study protocol. Contemporary Clinical Trials Communications 2019;14:100331.

12. Habich M, Letizia M. Pediatric Pain Assessment In the Emergency Department: A Nursing Evidence-Based Practice Protocol. Pediatr Nurs 2015;41:198-202.

13. Alzghoul BI. Pain Management Practices by Nurses: An Application of the Knowledge, Attitude and Practices (KAP) Model. Global Journal of Health Science 2015;8:154-60.

14. Arends RY, Bode C, Taal E. The role of goal management for successful adaptation to arthritis. Patient Education and Counseling 2013;93:130-8.

15. Resnick B. Pain management: The combined patient and provider perspective. Geriatr Nurs 2017;38:274-5.

16. Continuous quality improvement for nutritional care services in nursing homes: the importance of direct observation. Journal of the American Medical Directors Association 2006;7:61-2.

17. Martin-Saavedra JS, Vergara-Mendez LD, Pradilla I, et al. Standardizing music characteristics for the management of pain: A systematic review and metaanalysis of clinical trials. Complementary Therapies in Medicine 2018;41:81-9. 